\title{
Virtual Replicas for Remote Assistance in Virtual and Augmented Reality
}

\author{
Ohan Oda Carmine Elvezio Mengu Sukan \\ Steven Feiner \\ Dept. of Computer Science, Columbia University \\ New York, NY, 10027 USA \\ \{oo2116, ce2236, ms3774, skf1\}@ columbia.edu
}

\author{
Barbara Tversky \\ Dept. of Human Development, \\ Teachers College \\ New York, NY, 10027 USA \\ btversky@stanford.edu
}
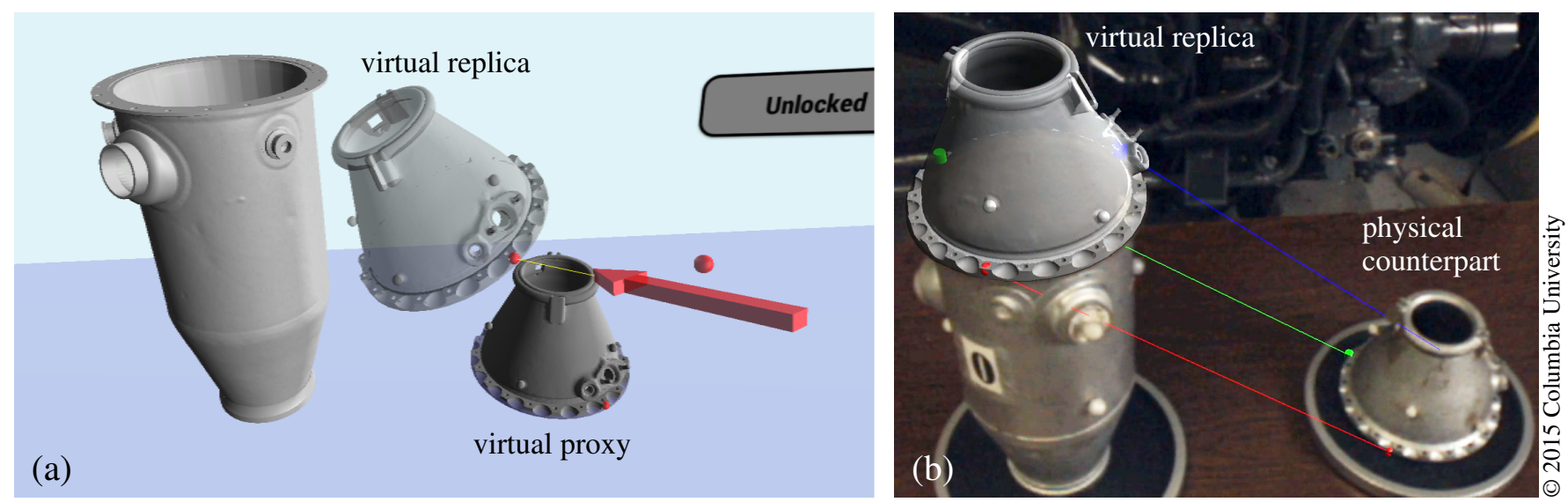

Figure 1. Two approaches allowing a subject-matter expert (SME) to guide a local user to place the top of an aircraft engine combustion chamber relative to its bottom by interacting with a virtual replica of the top. (a) PoINT3D, as seen by the SME in VR: The SME places 3D annotations (red spheres) on the transparent virtual replica, specifying contact points. The same annotations appear on the opaque virtual proxy corresponding to the virtual replica. (b) DEMO3D, as seen by the SME in AR, looking through the local user's cameras: The SME has placed the transparent virtual replica on the chamber bottom. Pairs of metaobject annotations on the virtual replica and physical chamber top are linked with color-coded lines to show the correspondence between the physical top and its virtual replica, and are also seen by the local user.

\begin{abstract}
In many complex tasks, a remote subject-matter expert may need to assist a local user to guide actions on objects in the local user's environment. However, effective spatial referencing and action demonstration in a remote physical environment can be challenging. We introduce two approaches that use Virtual Reality (VR) or Augmented Reality (AR) for the remote expert, and AR for the local user, each wearing a stereo head-worn display. Both approaches allow the expert to create and manipulate virtual replicas of physical objects in the local environment to refer to parts of those physical objects and to indicate actions on them. This can be especially useful for parts that are occluded or difficult to access. In one approach, the expert points in $3 \mathrm{D}$ to portions of virtual replicas to annotate them. In another approach, the expert demonstrates actions in 3D by manipulating virtual replicas, supported by constraints and annotations. We performed a user study of a 6DOF alignment task, a key operation in many
\end{abstract}

Permission to make digital or hard copies of all or part of this work for personal or classroom use is granted without fee provided that copies are not made or distributed for profit or commercial advantage and that copies bear this notice and the full citation on the first page. Copyrights for components of this work owned by others than ACM must be honored. Abstracting with credit is permitted. To copy otherwise, or republish, to post on servers or to redistribute to lists, requires prior specific permission and/or a fee. Request permissions from Permissions@ acm.org

UIST'15, November 08-11, 2015, Charlotte, NC, USA

(C) 2015 ACM. ISBN 978-1-4503-3779-3/15/11 ..\$15.00

DOI: http://dx.doi.org/10.1145/2807442.2807497 physical task domains, comparing both approaches to an approach in which the expert uses a 2D tablet-based drawing system similar to ones developed for prior work on remote assistance. The study showed the 3D demonstration approach to be faster than the others. In addition, the 3D pointing approach was faster than the $2 \mathrm{D}$ tablet in the case of a highly trained expert.

\section{ACM Classification Keywords}

H.5.m. Information Interfaces and Presentation: Multimedia Information Systems Artificial, Augmented, and Virtual Realities; I.3.6. Computer Graphics: Methodology and Techniques - Interaction Techniques; K.4.3. Computers and Society: Organizational Impacts - Computer-Supported Collaborative Work

\section{Author Keywords}

Collaborative mixed/augmented reality; 3D referencing techniques; remote task assistance; remote guidance; assembly; maintenance and repair.

\section{INTRODUCTION}

Task guidance has been an active topic in the field of augmented reality (AR), with applications to a wide range of domains, including the operation, assembly, maintenance, 
and repair of equipment (e.g., [3, 28, 5]). Seeing instructional graphics overlaid directly on the actual task environment can significantly improve a user's understanding compared to viewing instructions displayed on a nearby monitor or in a paper manual [8]. One especially productive approach to task guidance involves a remote subject-matter expert (SME) assisting a local user. Traditional approaches to remote guidance using voice or video limit how the SME can instruct the local user, especially for operations that require 3D spatial referencing and action demonstration. Language describing spatial locations and actions in space is frequently ambiguous or vague [26], leading to confusion and error (e.g., [7]). In contrast, AR enables a SME to directly interact with the local environment for 3D spatial referencing and action demonstration and allows a local user to visualize instructions directly overlaid on the environment.

Existing literature has explored approaches to enable SMEs to present instructional elements such as 3D arrows [4, 3], to perform hand gestures [24, 28], and to place annotations such as 3D tags or sketches on physical objects [14, 1, 9, 5]. However, it can be challenging or even impossible for a SME to refer to a part of physical object in the local user's environment that is occluded or to demonstrate actions on it.

We have developed two 3D interaction and visualization techniques for tasks that require more than simple pointing or annotation. Inspired by Voodoo-doll interaction [17], we developed approaches that allow the SME to create a virtual replica of its physical counterpart present in the local user's environment (Figure 1). The SME can then point to a location on the virtual replica and present that to a local user in AR (PoINT3D) (Figure 1a). Alternatively, the virtual replica can be used to demonstrate how to position and orient the physical counterpart (DEMO3D) (Figure 1b). However, it can be timeconsuming and error-prone to position and orient the virtual replica precisely due to interpenetration between the virtual replica and other objects in the local user's environment. To alleviate this problem, we use constraints to allow fast and precise placement of the virtual replica. We also display a set of metaobjects on a virtual replica and its physical counterpart, as shown in Figure 1(b), to help the local user mentally map the position and orientation of the virtual replica to its physical counterpart.

In addition, we provide the SME with two different perspectives on the local environment within which to interact, similar to Tatzgern et al. [27], who allow the user to switch between a live video and synthesized views of a reconstructed physical scene. Our first perspective is a virtual representation (Figure 1a) that uses virtual models of the 6DOF-tracked physical objects. The SME can freely navigate among and interact with these virtual models, whose poses can be efficiently transmitted. However, some virtual models may not correspond exactly to their physical counterparts because of damage or modification. In addition, it is sometimes necessary for the SME to be able to see exactly what is taking place in the local user's environment, from the perspective of the local user. To address these situations, we provide a second perspective that allows the SME to see and interact from the local user's live or frozen stereo camera views (Figure 1b).

Our work makes the following contributions: We develop two $3 \mathrm{D}$ interaction and visualization approaches in which a remote SME creates and uses virtual replicas of physical objects to guide a local user performing a task with those objects. The remote SME can switch seamlessly between a mirrored virtual reality (VR) representation of a local user's tracked environment, and shared live or frozen AR views from the perspective of the local user. These approaches (DEMO3D and POINT3D) combine principles from existing literature to efficiently support interaction in remote assistance scenarios, where both users wear tracked HWDs. We compare these approaches for a 6DOF alignment task (a core component of many remote-guidance scenarios) with 2D sketchbased annotation using a tablet (SKETCH2D) on speed of performance, ease of interaction by the remote SME, ease of interpretation by the local user, and preference. Our user study shows that DEMO3D was faster than both other approaches and POINT3D was faster than SKETCH2D with a highly trained SME. SMEs generally felt that they performed faster and better with DEMO3D than with SKETCH2D. Furthermore, local users preferred DEMO3D to SKETCH2D.

\section{PREVIOUS WORK}

Many researchers have explored approaches for supporting remote task guidance. Kuzuoka [13] showed that a SME can use their finger to indicate regions of interest in the video sent from a local user, with the composite imagery of the finger on the video sent back to the local user's display. Bauer et al. [2] extended this work to AR by presenting a 2D mouse cursor operated by the SME on the local user's head-worn display (HWD). However, the 2D pointer becomes outdated if the local user moves. To address this, Bottecchia et al. [3] allowed a SME to take a snapshot of the local user's view, and perform 2D interactions with the still image. However, pointing or gesturing on the local user's 2D view plane has limitations for conveying complex 3D actions. To allow demonstrations directly in the 3D environment, Kirk and Fraser [10] embedded the gestural output of a SME captured from an overhead video camera on a 3D plane where instructions are given in the local user's environment, while Goto et al. [6] presented a similar technique using prerecorded video. While these techniques may work well for tasks performed on a flat surface, they are not well suited for fully 3D tasks.

Chastine et al. [4] allow a SME to manipulate a 3D virtual arrow on a local user's view; however, it is difficult and timeconsuming to align the arrow. Bottecchia et al. [3] make it possible for a SME to insert a precomputed 3D virtual animation into a local user's view to demonstrate how to perform a task; however, a precomputed animation is not flexible enough to document actions that change based on the situation. Stafford et al. [24] devised a more flexible mechanism allowing a SME to reference a point of interest at a remote site. An indoor SME sees a virtual overhead view of a local user's world on a tabletop display and can point at the display directly to refer to a point of interest. This creates a virtual $3 \mathrm{D}$ representation of the indoor SME's hand from a texture- 
mapped visual hull determined by a set of cameras surrounding the tabletop. The representation of the hand is overlaid on the view of an outdoor user. Later, Tecchia et al. [28] use depth sensors to dynamically capture both the local user's environment and SME's hands. The 3D scene of the local user and the $3 \mathrm{D}$ hands of the SME are then merged and presented to both sides, allowing the SME to provide gestural instructions directly in the local user's environment. Sodhi et al. [23] applied this approach to a portable system. Although it can be easy to demonstrate simple actions with these techniques, it can be challenging to show how to precisely maneuver physical objects in the local user's environment: while the SME hand gestures are captured, the SME cannot manipulate the objects.

Kurata et al. [11] developed a system in which a local user wears a camera and a laser pointer on the shoulder. The SME sees the task space through the camera on the local user's shoulder, and references a point of interest in the physical environment with the laser. Sakata et al. [21] built on this work by using a chest-worn display and the capability for the SME to add line drawings on a still image captured from the local user's view. Lanir et al. [14] further extended this to a portable projector situated in the local user's environment, allowing a SME to add projected 2D drawings to the local user's physical environment. A video camera and a pico-projector are mounted at the end of a remotely-operated robotic arm so that they can be moved by a SME, who can interact with the live video feedback. In addition to sketching, Adcock et al. [1] allow the SME to manipulate preconstructed 2D proxies of physical objects in the local user's environment. The SME manipulates these proxies on a multi-touch display with gestures specifying 2D translations and rotations projected onto the local user's environment with a ceiling-mounted projector. However, projection-based approaches have limitations on pointing or drawing on complex surfaces and surfaces that are not directly projected.

To overcome these limitations, Kim et al. [9] presented a system that allows the SME to place annotations anywhere on the local user's physical environment, constructed dynamically with a SLAM (Simultaneous Localization and Mapping) algorithm. Gauglitz et al. [5] supplemented this technique by allowing the SME to freely navigate in the constructed scene to place annotations from a separate viewpoint in the local user's perspective. The constructed scene is updated dynamically to reflect the changes in the local user's environment. Although this technique would suffice for most guidance tasks, simple annotations on the physical environment cannot easily demonstrate actions such as 6DOF alignment, especially on complex surfaces.

Of the preceding approaches, only Adcock et al. [1] allow the SME to manipulate proxies, albeit entirely in 2D. Tait and Billinghurst [25] extend this so that a SME with a monoscopic desktop interface uses 2D input devices to specify a target layout for the local user by placing existing virtual copies of physical objects into a 3D model of the environment seen on the local user's HWD. In contrast, we wanted to allow the SME to view the local user's environment in a stereo HWD, while directly creating and manipulating, in 3D, replicas of tracked physical objects to demonstrate actions in the local user's environment.

\section{OUR APPROACHES}

Our approaches are based on the ways a SME guides a novice when co-located, by pointing to relevant places or demonstrating appropriate actions. In our approaches, the SME can create, annotate, and manipulate in 3D virtual replicas of physical counterparts in the local user's environment. This is inspired by voodoo-doll interaction [17], in which a user creates copies of existing virtual objects. The user can perform operations using the copies, which affect the original virtual objects. We employ a similar concept, adapted to multi-user remote assistance for ease of interaction by the SME and ease of interpretation by the local user.

As presented here, we assume that the physical objects with which the local user interacts have been modeled and are tracked in 6DOF. Even though it is possible to dynamically construct and track the local user's environment on the fly $[15,27,5]$, this can require a wide range of perspectives, obtained while the task objects are being manipulated, to allow proper segmentation. Additionally, parts not already in the local user's environment cannot be modeled, preventing the SME from referring to them. Thus, our implementation assumes the existence of 3D models of the necessary parts and a suitable technology to track them. However, some of these constraints can be lifted, as we describe later in the Discussion section.

We assume that the SME's environment contains a virtual proxy for each relevant physical object in the local user's environment and that the position and orientation of each virtual proxy is determined by the corresponding position and orientation of the physical object in the local user's environment. The SME can create a virtual replica of a physical object by grabbing its virtual proxy. The virtual replica can then be directly manipulated in 6DOF, remaining where the grab is released, and grabbed again to manipulate it further. The virtual replicas are also displayed to the local user in context of their physical counterparts.

We developed two approaches that take advantage of virtual replicas: POINT3D and DEMO3D. We use these approaches to guide placement of a physical object $A$ relative to another physical object $B$, a task found in many domains using remote assistance, including assembly, maintenance and repair.

\section{Point3D}

One way in which a physically co-located SME can guide a worker through an assembly task is by pointing to prominent landmarks on the objects and instructing the user to align them. For example, when assembling furniture, a SME might point to a peg on one part and a hole on another part, and say "That peg needs to be inserted into this hole." Specifying three pairs of contact points would allow the SME to convey the 6DOF pose of $A$ relative to $B$ (Figure 2a). In our implementation, the remote SME manipulates a tracked pointing device that controls a ray whose intersection with an object defines a contact point. After optionally creating one or more 

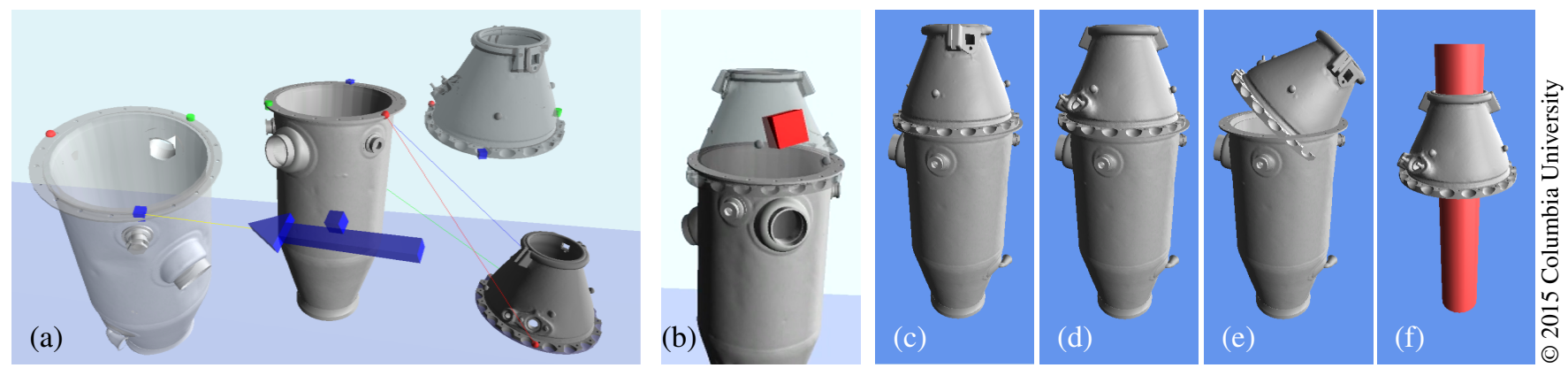

Figure 2. (a) PoINT3D: Once the SME specifies corresponding contact points on both objects, a color-coded rubberband line connecting the points appears. (b) DEMO3D: When the SME places and fine-tunes the chamber top relative to the bottom, the two can interpenetrate or not fit properly, as shown here, because there is no force feedback. (c-d) To restrict the top to 1DOF rotation along its "up" axis, a SME can define a constraint by placing the top above the bottom and indicating it can rotate only about its "up" axis, which allows these configurations. (e) However, this configuration would be prevented. (f) Alternatively, the SME can constrain the top to slide along and rotate around the red cylinder, restricting it to 2DOF motion.

virtual replicas, the SME can point to a location on either a virtual replica or its virtual proxy to place annotations anywhere on the corresponding physical object. The SME can freely manipulate a virtual replica to find a suitable pose from which to place an annotation. Annotations appear on both the virtual proxy and the virtual replicas in the SME's view and on the physical object in the local user's view. Since all annotations are attached to their objects, they are updated interactively as the objects are manipulated. Once annotations for corresponding points have been placed on both $A$ and $B$, a "rubberband" line will appear between corresponding annotations on both objects to help the local user identify which annotated points should be aligned (Figure 3 ).

\section{Demo3D}

An alternative approach allows the SME to use a virtual replica to directly show how to move to the final 6DOF pose of $A$ relative to $B$ in the local user's environment. Continuing our furniture assembly example for a physically co-located SME, this approach would be equivalent to the SME actually picking up a part, physically aligning it relative to another part to show the local user how the parts fit, and then placing it back, so that the local user can imitate the demonstrated action. One issue that arises in our case is that the virtual replica of $A$ can interpenetrate $B$ (Figure $2 \mathrm{~b}$ ), which can potentially

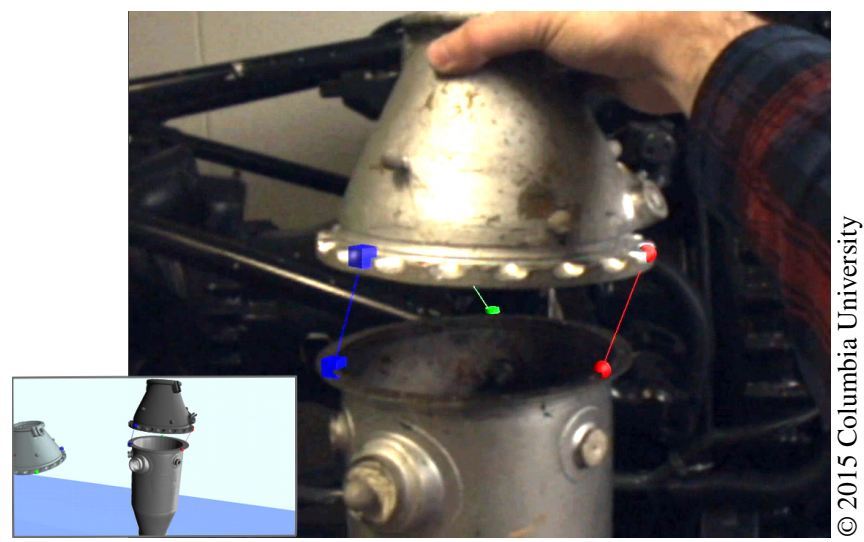

Figure 3. Point3D: The local user places chamber top on chamber bottom by matching the three contact points. (Inset shows SME's view.) cause misinterpretation. Prior work has used a physics simulation to avoid interpenetration and allow realistic interaction [18]. However, this can be computationally expensive and even cause unwanted repulsion to avoid interpenetration, as well as sliding and dropping in response to simulated gravity.

To address this, we employ a constraint-based approach similar to that of Adcock et al. [1], who constrain physical objects to flat surfaces. However, we use constraints that are more flexible and can be specified by the SME prior to giving guidance. In many real assembly tasks, two rigid parts fit together in a constrained way with some leeway in translation or orientation (Figure 2c-e). Thus, we allow the SME to orchestrate a set of rigid-body constraints, prior to guiding the local user, by placing the virtual replica at a location (or a region for a translational constraint) and specifying the DOFs the virtual replica can have (Figure 2f). When giving guidance, the SME can fine-tune the position and/or orientation within the DOFs allowed. This constraint-based approach not only prevents unwanted interpenetration, but also reduces potential manipulation errors introduced while fine-tuning the final 6DOF pose near the constrained region (Figure $2 b$ ).

Once the SME has specified the final 6DOF pose of the virtual replica, the local user must determine how to place the physical counterpart to match the pose. This raises another issue: the local user may have difficulty understanding how to match the physical object to the virtual replica because of difficulties performing the mental rotation. Considerable research has shown that mental rotation is difficult to imagine and that physical rotation facilitates mental rotation (e.g., [31]). Matching can also be difficult if the physical counterpart does not have enough prominent geometric features. To address this, we added a set of landmark metaobjects (Figure 1b) on the virtual replica, which are duplicated on its physical counterpart, with a connecting line between each metaobject and its duplicate to further simplify the matching process. The local user can use these metaobjects as cues to match the 6DOF pose of the physical counterpart with the virtual replica placed by the SME. Furthermore, we fade out any virtual replica as its physical counterpart gets within a preset threshold distance to the virtual replica placed by the SME, while maintaining the visibility of the metaobjects. We decided to fade out just the virtual replica, but not the metaobjects, since 


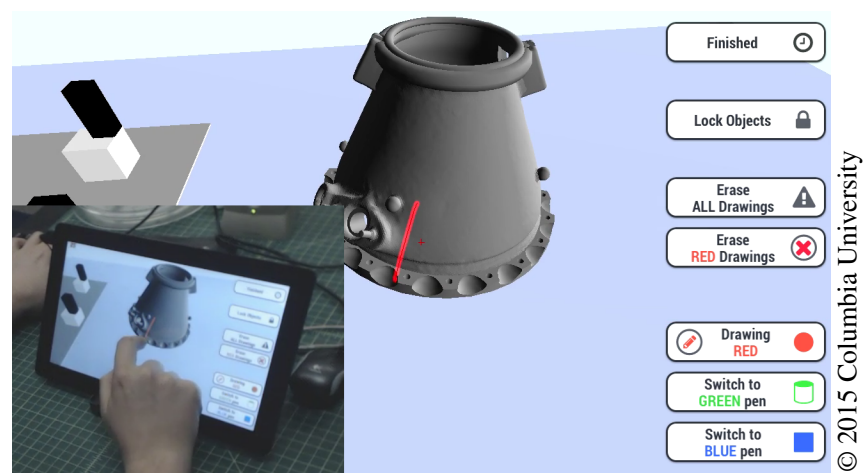

Figure 4. SKETCH2D: The SME sketches on corresponding contact points with multi-touch interaction. (Inset shows third-person view.)

$z$-buffer conflicts can occur as the physical counterpart overlaps with its virtual replica, making it visually confusing for the local user to fine-tune the final 6DOF pose. The appearance of the metaobjects themselves is intended to be sufficient for the local user to match the pose between the physical counterpart and its virtual replica when they are sufficiently near each other.

\section{Sketch2D}

As described previously, several recent remote task guidance systems provide the SME with a 2D annotation system [14, 1, $9,5]$ based on a multi-touch tablet or PC, in some cases with a projector in the local user's environment to project the SME's instructions onto the environment $[14,1,9]$. We developed a similar system that allows sketch-based 2D annotations on a multi-touch tablet for the SME (Figure 4), and displays the annotations on the local user's environment through a HWD. The SME uses multi-finger gestures to navigate among and draw annotations on virtual proxies. Each point sketched on the tablet screen is projected from the center of projection onto the closest point on the surface of the proxy object visible at that pixel, such that the sketches appear in $3 \mathrm{D}$ on the surfaces of the proxies.

\section{INTERACTION ENVIRONMENT}

For both the HWD and the tablet, the SME can transition seamlessly between two views without affecting what the local user sees: a VR view (Figure 1a) and an AR view (Figure 1b). In both views, the SME can grab (but not move) the virtual proxies to create virtual replicas, where the manipulation of the virtual replicas is presented to the local user. In the VR view, the SME is presented with a virtual environment, viewed from their own perspective, that includes virtual proxies of important tracked objects from the local user's environment, whose position and orientation are updated in real time as the local user manipulates them. Communicating geometric transformations, rather than video, between sites, minimizes network latency and avoids restricting the SME to views seen by the local user.

In the AR view, the SME interacts with camera imagery captured from the perspective of the local user. This can be useful when the SME wants to see the task environment from the local user's point of view, and when the task environment may contain unmodeled objects or objects that might not correspond to their models (e.g., if the objects have been damaged). The SME can also freeze the view (for the SME only) [3] to support interaction with the physical objects at their positions and orientations when the view was frozen. This is especially useful when the local user is moving their head or a physical object in a way that confuses the SME.

\section{IMPLEMENTATION}

Our experimental software is built using Goblin XNA [16] The SME creates the virtual replica of a virtual proxy by intersecting a 6DOF tracked manipulation device with the proxy and pressing a button. The manipulation device is rendered with a simple cubic box in the VR environment. As long as the button is depressed, the virtual replica remains rigidly attached to the manipulation device and can be manipulated freely. When the button is released, the virtual replica stays at the position and orientation at the time of release. The SME can grab and manipulate the virtual replica as many times as they want. If the SME instead grabs the virtual proxy, the previously created virtual replica of that proxy will disappear and a new virtual replica will be created. (Our implementation allows only a single virtual replica for each virtual proxy to reduce visual clutter.)

The 6DOF position and orientation of the physical objects in the local user's environment are tracked and streamed to the SME's system to update the virtual proxies. In addition, the 6DOF position and orientation of the SME's manipulation and pointing devices are streamed to the local user's system, and the 6DOF position and orientation of each user's head are streamed to the other user's environment, where they can be visualized, as we describe later.

\section{Point3D}

In POINT3D, the contact points on objects $A$ and $B$ are visualized as three metaobjects, each with a different shape and color, which protrude from the surface of each virtual proxy and virtual replica. We chose three contact points to support our rigid body manipulation tasks. Each pair of corresponding contact points on both objects is marked by metaobjects of the same shape and color. When instructing the local user, the SME first selects a metaobject, and then points to either a virtual replica or a virtual proxy to place it. The SME must place metaobjects on three contact points of each object to fully define the 6DOF pose of $A$ relative to $B$. Selection of a metaobject and point on an object is done using a 6DOF tracked pointing device, with two buttons to trigger pointing and choose a metaobject. We support bimanual interaction, in which the SME holds the manipulation device with her nondominant hand, and the pointing device with her dominant hand (Figure 5b).

Whenever the SME is pointing to an object, a 3D arrow appears in the local user's environment to help the local user identify the pointing pose. The arrow always appears relative to the physical object, since the local user cannot see the corresponding virtual replica. Initially, the technique placed the metaobject only when the SME placed the pointing device close enough to touch the surface without penetrating, but we 
found that without a physical obstruction preventing penetration of the object's surface, users would struggle to quickly place an annotation via ballistic pointing. To address this, we replaced the "touch and place" interaction with a raycast.

\section{Demo3D}

In DEMO3D, the SME uses the manipulation device in their dominant hand (Figure 5a) to create a virtual replica from its proxy and place the replica directly at the desired location. We make the virtual replica snap to the constrained location with a smooth transition when the SME releases the virtual replica near a region where a constraint is specified. The virtual replica will be left at the location at which it is released if there are no constraints within a threshold distance and orientation.

An arbitrary number of metaobjects are defined by the SME prior to the study, just like the constraints. Metaobjects can be added anywhere on the 3D model representing a physical object, preferably on prominent geometrical points, and will appear in the local user's environment as soon as the SME creates a virtual replica from a virtual proxy. The virtual replica fades out when the bounding boxes of the virtual replica and physical counterpart start to overlap, and fades in when the overlapping is resolved. To avoid fading out the replica while the SME is manipulating it, the fading behavior occurs only after the SME places the virtual replica.

\section{Sketch2D}

We implemented the following functionality with multifinger gestures on the tablet: sketching lines, translating the camera in three dimensions, panning and tilting the camera, translating the camera towards a tapped location, and resetting the camera to a default position and orientation. The SME can also orbit the camera around a point determined by the closest intersection between the central axis of the view volume and the model. In addition, we allow the SME to sketch using different colors and erase either all sketches or just those of a particular color. To enhance visibility, lines are drawn with a glow effect, as shown in Figure 4. In the AR view, the SME can sketch, but not navigate, since the SME cannot control the local user's perspective (except to freeze the SME's copy).

\section{PILOT STUDIES}

Prior to conducting the formal user study, we performed informal pilot studies with our lab members and 12 compensated participants.

Initially, the SME interacted using natural hand gestures tracked by a depth camera [30]. However, the limited tracking range prevented smooth interaction with the virtual replicas. To address this, we switched to a Nintendo Wii remote, equipped with optical markers, which can be tracked in a far larger volume. However, certain motions were difficult and time-consuming. In addition, one-handed interaction required POINT3D to take longer due to the sequential process of pointing after grabbing and manipulating. Therefore, our implementation is now bimanual, using an easy-to-hold pointing device and a device for grabbing and manipulating, both outfitted with optical markers.
When testing the DEMO3D and SKETCH2D techniques, we found that if the local user moved the physical parts before the SME completed instructing, the movement of the virtual proxy would interfere with completing the instructions. To avoid this, we introduced a "lock" feature that prevents tracking updates from affecting the SME's environment until unlocked. We provided the SME with a foot-pedal to toggle between locking and unlocking for POINT3D and DEMO3D. For SKETCH2D, the SME taps on a button on the screen to toggle.

We initially showed an animation interpolating between the pose of a physical part and its virtual replica in DEMO3D to help the local user mentally map the 6DOF pose from the virtual replica to its corresponding physical part. However, we found out that the animation was not helping the local user much. Instead, we discovered that contact point matching in POINT3D was easier for the local user to interpret. Therefore, we decided to replace the animation with a set of easily visible metaobjects on the physical part and virtual replica to further improve the performance of DEMO3D.

In certain trials, it was difficult for the SME to manipulate the virtual replicas without reorienting themselves relative to the local user's environment. However, our setup sometimes made it difficult for the SME to physically move to a desired vantage point. To alleviate this, we provided the SME with an orientation-tracked physical lazy susan turntable to rotate their virtual view relative to the local user's environment (Figure $5 \mathrm{a}$ ).

The SME did not need to view the local user's environment in AR for the tasks in our study, since the physical objects were represented by accurate virtual models; further, we found that none of the participants in our pilot studies intentionally switched from VR to AR. Therefore, we removed the ability to switch between VR and AR for the formal study to simplify the user interface.

\section{USER STUDY}

We conducted a formal user study to compare the performance of POINT3D, DEMO3D, and SKETCH2D. We required that the accuracy with which the local user performed each trial be within a small range of distance and orientation from correct pose for the trial to end; therefore, we compared only time, not accuracy. To emulate the voice communications that would be supported in a remote collaboration environment (e.g., [13]), we allowed the two participants to communicate verbally during the trials to clarify misunderstandings or describe subtle adjustments.

\section{Hypotheses}

Based on an analysis of the tasks and the pilot studies, we predicted:

H1. Demo3D should be faster than Point3D. We expect POINT3D to take longer because it requires the SME to make six annotations, as opposed to DEMO3D, which only requires a single motion for demonstration. DEMO3D also allows for a quicker/less precise alignment because of its embedded 5DOF constraint. 

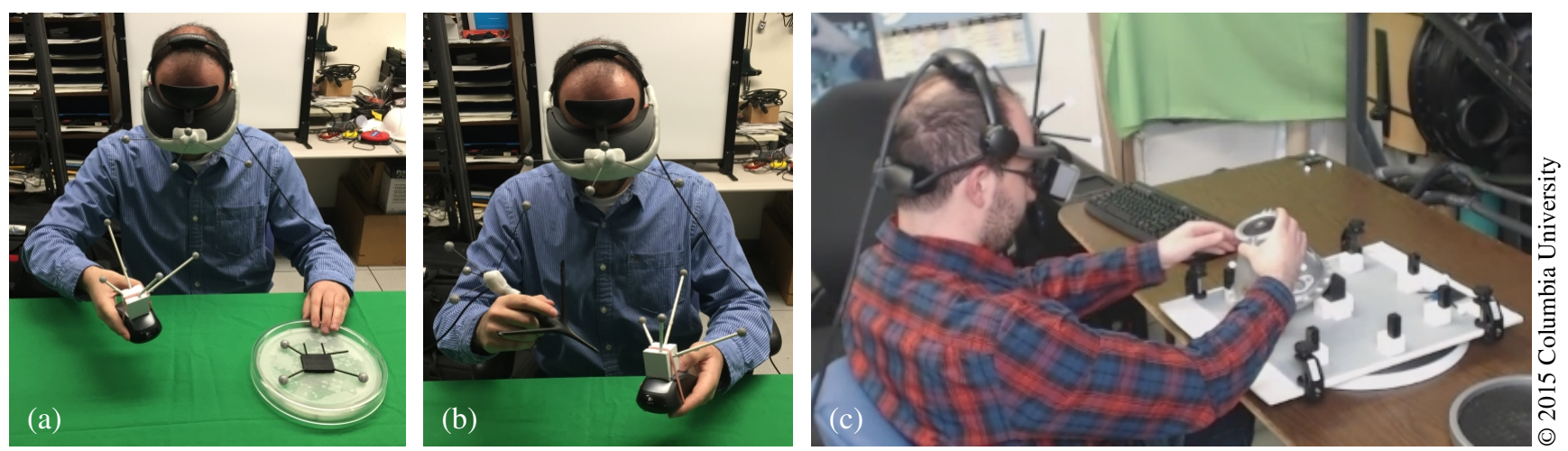

Figure 5. A SME wearing a tracked Sony HMZ-T3W HWD interacts with (a) a tracked mouse and lazy susan turntable in DEMO3D, and (b) a tracked Leonar3Do bird controller and mouse in PoINT3D. (c) A local user wearing a tracked Canon HM-A1 HWD places an aircraft engine combustion chamber top on a Lego fixture.

H2. Point3D should be faster than SKetch2D. Similar to PoINT3D, SKETCH2D also requires six annotations per alignment, but it additionally burdens the SME with 3D virtual camera navigation to be able to sketch on certain parts of objects. With POINT3D, the SME should be able to insert annotations relatively quickly using bimanual pointing. Both POINT3D and DEMO3D should require less interpretation time by the local user, compared to SKETCH2D, because of the assistance provided by the virtual rubberband lines that connect corresponding metaobjects.

H3. Both SMEs and local users should prefer DEMO3D. We expect this because DEMO3D should be quicker and less cognitively challenging for the same reasons provided for $\mathrm{H} 1$ and $\mathrm{H} 2$.

The local user's task is inherently 3D: to translate and rotate the chamber top to the specified position and orientation. For the SME, demonstrating the action to be performed is a more direct way of communicating than finding and marking the points of contact. For the local user, seeing the action is more easily comprehended and performed than finding and matching the points of contact. Research on the mirror neuron system in the brain suggests direct connections between perceiving an action and performing it (e.g., [20]). Both methods are $3 \mathrm{D}$, as is the task, so both methods should surpass the 2D tablet method.

\section{Methods}

\section{Participants}

We recruited 22 participants from our institution (5 male), 18-26 years old (average 22), through email and posted flyers. Participants were recruited as dyads who attended a single-session experiment together. Two participants had previous experience with $A R$, and none had any familiarity with our techniques.

\section{Equipment}

In PoINT3D and DEMO3D, participants assigned to the role of SME wore a Sony HMZ-T3W opaque stereo HWD. In DEMO3D, the SME held a tracked mouse in their dominant hand and turned a lazy susan turntable with their nondominant hand (Figure 5a); in POINT3D, the SME held a tracked Leonar3Do bird controller in their dominant hand, and the tracked mouse in their non-dominant hand (Figure 5b). The HWD, mouse, bird controller, and lazy susan turntable were tracked by an 10-camera NaturalPoint OptiTrack V100 tracking system, running on a computer powered by an Intel i7-4770k with 16GB of RAM and an Nvidia GeForce GTX 770. A foot-pedal was placed under a table to perform the "lock" feature described earlier. SMEs used an untracked Samsung Series 7 Slate Tablet in SKETCH2D.

Participants assigned the role of local user wore a Canon HMA1 stereo video-see-through HWD, as shown in Figure 5(c), running on a computer powered by an Intel i7-3770k with 16GB of RAM and an Nvidia GeForce GTX 780. Local users interacted with two physical objects that were replicated digitally for the SME. One was the top part of an aircraft engine combustion chamber, and the second was a fixture created for our study from $2 \times 2$ Lego bricks and a Lego $48 \times 48$ baseplate, as shown in Figure 5(c). (We used the fixture instead of the chamber bottom of Figure 1 to support a far wider range of possible 6DOF poses for the study.) The HWD, aircraft engine combustion chamber, and Lego fixture were tracked by a 12-camera NaturalPoint OptiTrack S250E tracking system.

\section{Design}

Since the participant playing the role of the SME is not a real SME, it was essential that the system provide instructions to the study participant to guide them in their role. To make the instruction easy to interpret and equivalent for all conditions, we added visual hints, similar to the POINT3D metaobject annotations, showing three contact points on both the top and the Lego fixture. In POINT3D and SKETCH2D, the SME would use these contact points as a hint on how to place metaobject annotations or sketches. In DEMO3D, the SME would attempt to align the three corresponding contact points on each object. These contact points are visualized using the same shapes and colors used in POINT3D.

The instructional contact points for both objects are prepared before the study, to be shown by the system to the participant playing the role of the SME. In the informal pilot and the formal user study, the SME's goal was to place the top on the Lego fixture. The Lego fixture was organized as a ring of eight 3-level pegs with another 3-level, 4-sided peg at the center of the ring. Contact points for this fixture were defined 
as the exposed face of a Lego peg level. In total, there were 36 discrete contact points available for this fixture. Contact points on the top were defined in two phases. First, a set of contact point combinations are prepared for each unique, possible configuration relative to the Lego fixture. Three contact point combinations were chosen for this study. Second, the contact points are rotated about the center "up" axis of the chamber top. Since there were various protrusions on the top that would make certain poses difficult to match, we chose angles for the second phase that were achievable. In total, six angles were chosen for the second phase, representing ranges of acceptable angles truncated to $18^{\circ}$ offsets, which aligned with generic holes on the chamber flange.

There were three within-subject interaction techniques $\times 8$ ring pegs $\times 3$ peg combinations $\times 6$ relative yaw offsets $=$ 144 unique 6 DOF poses. Trials were blocked by technique and randomized by contact point combination and rotation angle for the top, and Lego fixture peg selection. Each block included three practice trials and six timed trials. Each technique described above was experienced first by one third of the dyads. The order of techniques was counterbalanced across dyads to minimize bias due to learning.

\section{Procedure}

Participants were welcomed by the study coordinators and given the PseudoIsochromatic Plate (PIP) Color vision test to screen for color blindness, the Stereo Optical Co. Inc. Stereo Fly Test (SFT) to screen for stereo vision, and the Vandenberg-Kuse Mental Rotation Test (MRT) [29] to screen for spatial ability. The dyad member scoring higher on the MRT was assigned the role of SME, to reduce the effect of low spatial ability on instruction preparation. All participants passed the SFT; one (assigned as a local user) had some difficulty with the PIP, but did not perform worse than others in that role. After completing the tests and being assigned roles, participants were introduced to the study and given role-specific instructions. Before each block, participants were given a detailed explanation of the interaction techniques. For SKETCH2D, an instruction sheet was provided to explain all possible controls. The participants were then shown a demo and allowed to explore the techniques.

The experiment had three segments, one for each technique, and each had two blocks. In the first block, one of the researchers served as SME, and the participant assigned the role of the local user played that role. In the second block, the participant assigned the role of SME played that role and the other participant continued as the local user. The first block was necessary to demonstrate how to perform as a SME.

At the start of each trial, the SME was shown the virtual proxies and semi-transparent instructional metaobjects over the contact points on each object, and instructed to convey to the local user the 6DOF pose using the current interaction technique. The SME was instructed to press a dedicated "finished" button (by either tapping on a UI button on the tablet, or intersecting the manipulation device with a virtual button placed next to the Lego fixture proxy) to signal to the local user that the instruction had been prepared. However, the local users were encouraged to begin moving the chamber top as soon as they felt they understood the instruction. Once the pose had been acceptably matched, the virtual proxy turned green. The acceptable range was set to be $25 \mathrm{~mm}$ in position and $7^{\circ}$ in orientation (summed over all three axes of rotation) based on our pilot study. As soon as the pose had been acceptably matched, the SME confirmed by pressing the same button, now labelled "confirm." Once the trial was complete, the local user returned the top to the starting position, and all virtual annotations and replicas were removed in preparation for the next trial. Throughout the study, the positions and orientations of the participants' heads, the manipulation device, the pointing device, the annotations, physical objects (and by extension, the virtual proxies), and virtual replicas were recorded.

Participants were asked to complete a three-part questionnaire before, during, and after the study, assessing the three techniques. The questionnaire included an unweighted NASA TLX and a request to rank the techniques from 1 ("Least Preferred") to 3 ("Most Preferred").

\section{RESULTS}

We analyzed overall completion time separately by whether the SME was played by a researcher (Trained SME) or a participant (Novice SME). Each condition had a total of 198 trials (11 dyads $\times 3$ conditions $\times 6$ timed trials). We identified outliers using Tukey's outlier filter, resulting in 5.6\% (11 of 198 trials: four DEMO3D, four POINT3D, three SKETCH2D) of Trained SME data and 6.1\% (12 of 198 trials: six DEMO3D, two PoINT3D, four SKETCH2D) of Novice SME data being excluded from the rest of our analysis. We evaluated our hypotheses for significance using a Bonferroni-corrected $\alpha$ of $0.0167(0.05 / 3)$.

We fit a linear mixed effects model to our data using R [19] to model completion time as a function of technique (fixed effect) and participant (random effect). Compared to a base model with only participant as a random effect, a KenwardRoger corrected F-test showed that technique was significant as a fixed effect for both Novice $\operatorname{SME}\left(F_{(2,173.106)}=\right.$ $\left.79.269, p<10^{-4}\right)$ and Trained SME $\left(F_{(2,173.91)}=153.82, p<\right.$ $10^{-4}$ ) (Figure 6a). A pairwise least-squares means comparison revealed that the Trained SME was significantly faster using DEMO3D than POINT3D $\left(t_{(178.44)}=-7.199, p<10^{-4}\right)$, validating $\mathrm{H} 1$. The Trained SME was also significantly faster using POINT3D than SKETCH2D $\left(t_{(177.75)}=-10.230, p<\right.$ $\left.10^{-4}\right)$, supporting H2. For the trials with a Novice SME, DEMO3D was significantly faster than POINT3D $\left(t_{(177.13)}=\right.$ $-11.944, p<10^{-4}$ ), validating H1. However, completion times between POINT3D and SKETCH2D did not differ significantly $\left(t_{(177.12)}=2.336, p=.0618\right)$, failing to support $\mathrm{H} 2$.

Recalling that only recruited participants received the questionnaire, all SME ratings and rankings are for Novice SMEs. A majority of participants (7 SMEs and 8 local users, $64 \%$ and $73 \%$ respectively) ranked DEMO3D as their most preferred technique (Figure 6b), supporting H3. A Friedman test conducted to determine whether participants had a differential rank ordered preference indicated a statistically significant differential preference between techniques for local 


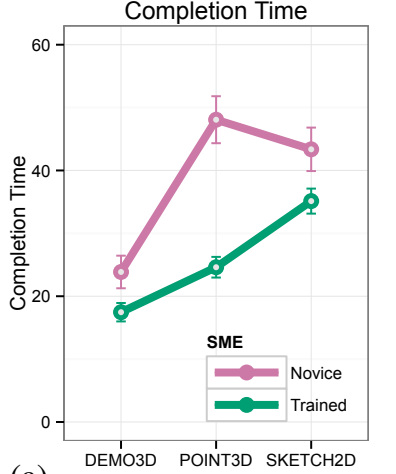

(a)

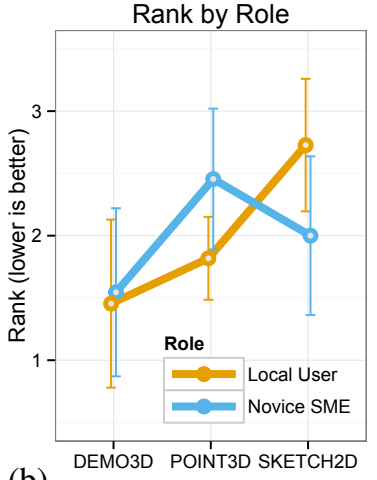

(b)
NASA TLX by Technique and Role

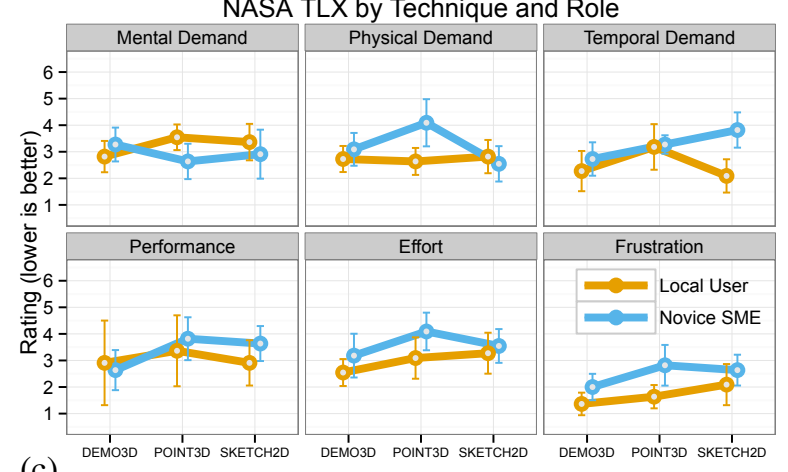

(c)

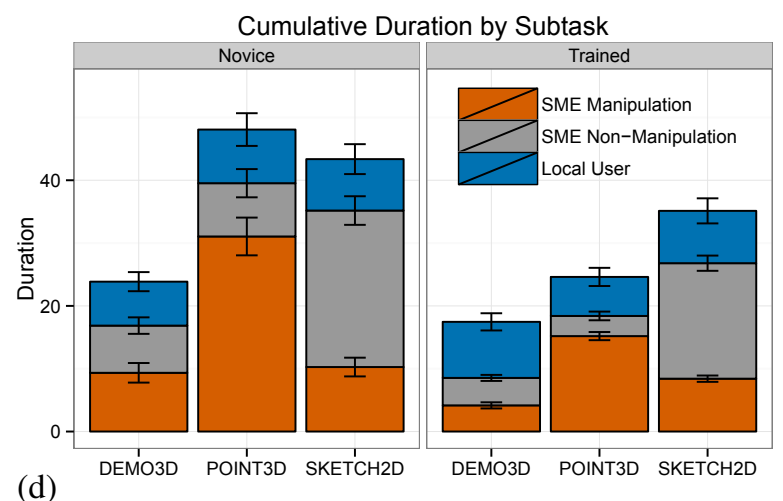

(d)

Figure 6. (a) Mean completion time in seconds for Novice and Trained SMEs. (Error bars show 95\% confidence intervals normalized to remove between-subject variability.) (b) Overall technique preference by role (1=most preferred, $3=$ =least preferred). (c) Unweighted NASA TLX survey. (d) Task duration breakdown for Novice and Trained SME.

users, $\chi_{(2)}^{2}=9.4545, p<.01$. A post hoc comparison using Nemenyi's procedure showed that rankings of DEMO3D were significantly more favorable than those for SKETCH2D, $p<.01$, partially validating $\mathrm{H} 3$ for local users. While local users on average ranked POINT3D higher than SKETCH2D and lower than DEMO3D, the difference between those two techniques was not significant. SMEs on average ranked DEMO3D first, SKetCH2D second, and Point3D last, but the resulting Friedman test statistic was not significant, $\chi_{(2)}^{2}=$ 4.5455, $p=.103$, supporting, but not validating, $\mathrm{H} 3$ for SMES.
Friedman tests applied to the unweighted NASA TLX survey results (Figure 6c) revealed a significant difference between techniques in terms of perceived physical demand, $\chi_{(2)}^{2}=9.1852, p=0.01$, temporal demand $\chi_{(2)}^{2}=6.75, p=$ 0.03 , and perceived performance, $\chi_{(2)}^{2}=6.8125, p=0.03$, by SMEs using an $\alpha$ of 0.05 . Post hoc comparisons using Nemenyi's procedure revealed that SMEs felt POINT3D was physically more difficult than SKETCH2D. This may be because many participants are already comfortable interacting with 2D multi-touch displays using relatively common gestures, as opposed to attempting bimanual 3D pointing in immersive VR. (Several participants commented that they were used to touchscreen interactions.) Another possibility could be that the bimanual $3 \mathrm{D}$ pointing task of POINT3D is more physically demanding. In addition, POINT3D might have been at a further disadvantage because SMEs could not see their hands directly, occasionally resulting in their 3D controllers colliding with each other or their HWD. The rest of the pairwise comparisons between techniques for SMEs were not statistically significant; however, it is interesting to note that SKETCH2D had the lowest (best) average rank for physical demand, while DEMO3D had the lowest (best) average rank for performance and temporal demand. For local users, there was no significant difference between techniques in the TLX survey results, with generally low task load reported across all techniques.

\section{DISCUSSION}

\section{Study Results}

Most of the task time was used by the SME. The approaches had a minimal effect on the time it took local users to place the top once instructed. Both Novice and Trained SMEs were faster using DEMO3D. DEMO3D took little training; Novice SMEs were barely slower than the Trained SMEs. We believe DEMO3D was faster and more preferred because showing how to place the top at the correct position and orientation is more direct and natural than finding and annotating contact points. A participant commented about DEMO3D that "This one is more human. The grabbing feels like manipulating things with my own hands with the gears", and another commented "Sometimes it is hard to put the object in the right place. But it is easy and entertaining to actually place the object." AR was key to creating transparent 3D virtual models that could be easily manipulated and visualized.

To understand why Novice SMEs did not perform better using POINT3D than SKETCH2D, as we had hypothesized (H2), we analyzed usage patterns for each technique by breaking down the overall completion time into subtasks. One of the reasons we expected SKETCH2D to perform worse than DEMO3D and POINT3D was the amount of virtual camera navigation required by SKETCH2D. To understand how participants actually spent their time interacting with our system, we divided the overall completion time into SME and local user time (i.e., time between the start of the trial and when the SME pressed the "finished" button to signal to the local user that their instructions were ready). We further divided SME time into manipulation and non-manipulation time. For SKETCH2D, manipulation time was defined as the period during which 
the SME was touching the screen either to draw or to navigate with the virtual camera. For POINT3D, manipulation time was accumulated whenever the SME grabbed, held, or pointed at a virtual replica. For DEMO3D, manipulation time was simply the amount of time the SME grabbed and held a virtual replica while placing it onto the virtual fixture.

The results of this breakdown are shown in Figure 6(d). Surprisingly, SME manipulation time was much faster for SKETCH2D than POINT3D, even though both require adding six annotations. However, inspection of the videos revealed that most of SME time in SKETCH2D was spent with the SME's fingers hovering over the screen, but not actually touching it. Based on our observations, we believe that this hovering behavior occurred mainly because a SME (1) when navigating and drawing, touches near the screen center and taps on-screen buttons placed near the screen edge to change sketch colors or proceed to the next step and (2) needs extra time to plan virtual camera navigation and recall the necessary gestures to execute those actions. For POINT3D, we were surprised by how much time and effort Novice SMEs needed for bimanual pointing. We believe this could be improved if SMEs could see their hands directly (in AR, rather than VR) and avoid accidental collision of tracked devices with one another and with the HWD.

Additionally, because holding the manipulation and pointing devices near the head or near each other resulted in degradation in tracking performance (causing overlap of retroreflective IR markers from the perspective of ceiling mounted cameras), we observed Novice SMEs having to hold unnatural poses for longer periods of time, which may have also contributed to their perception of increased physical demand over SKETCH2D. Alternative tracking technologies, including ones that do not require line-of-sight (e.g., electromagnetic, which could work well in the SME's purely virtual environment), could alleviate this issue by allowing the SME to sit in a comfortable pose (e.g., leaning back, elbows resting) and fully leverage the affordances of bimanual interaction.

\section{Generality of our Approach}

As described above, our techniques assume that manipulable physical objects have been modeled and are tracked in 6DOF. This can be practical in many controlled scenarios, as most items are now designed and manufactured using 3D CAD models, which could be used as virtual proxies. When that is not feasible, a dynamic scene reconstruction algorithm could be used (e.g., $[15,27,9,5])$. To ensure work objects can be distinguished from each other, they could be introduced one at a time and segmented by analyzing the differences between previous and current scans. Based on the particular setting, other tracking solutions could replace OptiTrack (e.g., feature-based or model-based vision, Valve Lighthouse, or SLAM).

Our approach can also be applied in a partially tracked and modeled environment. DEMO3D already allows a SME to demonstrate how to fit a single modeled and tracked part (e.g., the chamber top) into a static unmodeled environment, albeit without constraints. Because the SME can elect to see the local user's environment in stereo AR (Figure 1b), they can direct the local user to look at specific places in the environment and demonstrate using the virtual replica of the single tracked and modeled part relative to the static environment, relying on depth cues from the stereo image pair. POINT3D could also be used in such an environment with a small modification: Adding annotations is currently done by pointing (with raycasting to the closest intersection), requiring a modeled environment; instead, raycasting could be easily be replaced with direct 3D placement. Alternatively, if the local user's HWD includes a depth camera, POINT3D could work unmodified.

DEMO3D uses constraints to ease instruction for the SME and pre-generated metaobjects to provide visual clues to the local user. In a dynamically modeled scene, the SME might define constraints by demonstration using virtual replicas or a physics engine could be used to apply gravity to or avoid interpenetration between objects. To minimize SME preparation time prior to guidance, metaobjects can be automatically generated at prominent geometrical feature points (e.g., using Harris3D [22]). We also note that defining constraints is optional, even though it is a simple, one-time process that is especially beneficial where similar instructions are provided repeatedly (e.g., to different local users).

\section{CONCLUSIONS AND FUTURE WORK}

We presented two approaches (DEMO3D and POINT3D) in which a remote SME, using virtual replicas of physical objects, guides a local user performing a 6DOF alignment task. We compared these approaches with a 2D sketch-based approach (SKETCH2D) similar to ones used in previous work. A user study showed that DEMO3D performed faster than the other approaches, and POINT3D performed faster than SKETCH2D with a highly trained SME. DEMO3D was preferred over SKETCH2D by local users, and SMEs generally felt DEMO3D was faster and better than SKETCH2D.

Our study used tracked devices for manipulation and pointing. As briefly discussed in our Pilot Study section, we initially implemented bimanual interaction using hand-tracking. Once hand-tracking with improved stability and range becomes available, both DEMO3D and POINT3D can be easily adapted to use bare hands for more natural interaction.

Our techniques currently target defining a $6 \mathrm{DOF}$ pose of $A$ relative to $B$. However, DEMO3D can be extended to demonstrate successive steps to move $A$ from one location to another using more complex maneuvers by providing a demonstration similar to that described in our Pilot Study section, but with an exact trace of the SME's actions.

Finally, having the SME be telepresent in the local user's environment through full-body reconstruction [12] could make our techniques applicable to a broader range of tasks involving more than just manual interaction.

\section{ACKNOWLEDGMENTS}

This research was funded in part by NSF Grants IIS-0905569 and IIS-0905417. Canon U.S.A. Inc. generously lent the HMA1 HWD. Leonar3Do donated the bird controller. Goblin XNA was developed in part through funding from Microsoft 
Research and NSF. We thank Steve Henderson for refurbishing the aircraft engine combustion chamber used in the study.

\section{REFERENCES}

1. M. Adcock, D. Ranatunga, R. Smith, and B.H. Thomas. 2014. Object-Based Touch Manipulation for Remote Guidance of Physical Tasks. In Proc. ACM SUI. 113-122.

2. M. Bauer, G. Kortuem, and Z. Segall. 1999. "Where Are You Pointing At?" A Study of Remote Collaboration in a Wearable Videoconference System. In Proc. IEEE ISWC. 151-158.

3. S. Bottecchia, J. Cieutat, and J. Jessel. 2010. T.A.C: Augmented Reality System for Collaborative Tele-assistance in the Field of Maintenance Through Internet. In Proc. ACM AH. 14:1-14:7.

4. J. Chastine, K. Nagel, Y. Zhu, and M. Hudachek-Buswell. 2008. Studies on the Effectiveness of Virtual Pointers in Collaborative Augmented Reality. In Proc. IEEE 3DUI. 117-124.

5. S. Gauglitz, B. Nuernberger, M. Turk, and T. Höllerer. 2014. World-Stabilized Annotations And Virtual Scene Navigation For Remote Collaboration. In Proc. ACM UIST. 449-459.

6. M. Goto, Y. Uematsu, H. Saito, S. Senda, and A Iketani. 2010. Task Support System by Displaying Instructional Video Onto AR Workspace. In Proc. IEEE ISMAR. 83-90.

7. J. Heiser, B. Tversky, and M. Silverman. 2004. Sketches for and from Collaboration. Visual and spatial reasoning in design III 3 (2004), 69-78.

8. S. Henderson and S. Feiner. 2011. Augmented Reality in the Psychomotor Phase of a Procedural Task. In Proc. IEEE ISMAR. 191-200.

9. S. Kim, G. Lee, N. Sakata, and M. Billinghurst. 2014. Improving Co-Presence with Augmented Visual Communication Cues for Sharing Experience through Video Conference. In Proc. IEEE ISMAR. 83-92.

10. D.S. Kirk and D.S. Fraser. 2005. The Effects of Remote Gesturing on Distance Instruction. In Proc. CSCL. 301-310.

11. T. Kurata, N. Sakata, M. Kourogi, H. Kuzuoka, and M. Billinghurst. 2004. Remote Collaboration Using a Shoulder-Worn Active Camera/laser. In Proc. IEEE ISWC, Vol. 1. 62-69.

12. G. Kurillo, R. Bajcsy, K. Nahrsted, and O. Kreylos. 2008. Immersive 3D Environment for Remote Collaboration and Training of Physical Activities. In Proc. IEEE VR. 269-270.

13. H. Kuzuoka. 1992. Spatial Workspace Collaboration: A SharedView Video Support System for Remote Collaboration Capability. In Proc. ACM CHI. 533-540.

14. J. Lanir, R. Stone, B. Cohen, and P. Gurevich. 2013. Ownership and Control of Point of View in Remote Assistance. In Proc. ACM CHI. 2243-2252.
15. R.A. Newcombe, S. Izadi, O. Hilliges, D. Molyneaux, D. Kim, A.J. Davison, P. Kohli, J. Shotton, S. Hodges, and A. Fitzgibbon. 2011. KinectFusion: Real-time Dense Surface Mapping and Tracking. In Proc. IEEE ISMAR. 127-136.

16. O. Oda and S. Feiner. 2015. Goblin XNA Framework. (2015). http://goblinxna. codeplex.com/

17. J.S. Pierce, B.C. Stearns, and R. Pausch. 1999. Voodoo Dolls: Seamless Interaction at Multiple Scales in Virtual Environments. In Proc. ACM i3D. 141-145.

18. T. Piumsomboon, D. Altimira, H. Kim, A. Clark, G. Lee, and M. Billinghurst. 2014. Grasp-Shell vs Gesture-Speech: A Comparison of Direct and Indirect Natural Interaction Techniques in Augmented Reality. In Proc. IEEE ISMAR. 73-82.

19. R Core Team. 2015. R: A Language and Environment for Statistical Computing. http://www. R-project. org/

20. G. Rizzolatti and L. Craighero. 2004. The Mirror-Neuron System. Annu. Rev. Neurosci. 27 (2004), 169-192.

21. N. Sakata, T. Kurata, and H. Kuzuoka. 2006. Visual Assist with a Laser Pointer and Wearable Display for Remote Collaboration. In Proc CollabTech. 66-71.

22. I. Sipiran and B. Bustos. 2011. Harris 3D: A Robust Extension of the Harris Operator for Interest Point Detection on 3D Meshes. Vis. Comput. 27, 11 (2011), 963-976.

23. R.S. Sodhi, B.R. Jones, D. Forsyth, B.P. Bailey, and G. Maciocci. 2013. BeThere: 3D Mobile Collaboration with Spatial Input. In Proc. ACM CHI. 179-188.

24. A. Stafford and W. Piekarski. 2008. User Evaluation of God-like Interaction Techniques. In Proc. AUIC. 19-27.

25. M. Tait and M. Billinghurst. 2014. View independence in remote collaboration using AR. In Proc. IEEE ISMAR. 309-310.

26. L. Talmy. 2003. Toward a Cognitive Semantics. Vol. $1-2$. MIT press.

27. M. Tatzgern, R. Grasset, D. Kalkofen, and D. Schmalstieg. 2014. Transitional Augmented Reality Navigation for Live Captured Scenes. In Proc. IEEE VR. 21-26.

28. F. Tecchia, L. Alem, and W. Huang. 2012. 3D Helping Hands: A Gesture Based MR System for Remote Collaboration. In Proc. ACM VRCAI. 323-328.

29. S.G. Vandenberg and A.R. Kuse. 1978. Mental Rotations, a Group Test of Three-dimensional Spatial Visualization. Perceptual and motor skills 47, 2 (1978), 599-604.

30. R. Wang, S. Paris, and J. Popović. 2011. 6D Hands: Markerless Hand-tracking for Computer Aided Design. In Proc. ACM UIST. 549-558.

31. M. Wexler, S.M. Kosslyn, and A. Berthoz. 1998. Motor Processes in Mental Rotation. Cognition 68, 1 (1998), 77-94. 